\title{
Nonlinear Silicon Nanophotonics for Mid-Infrared Applications
}

\author{
William M. J. Green ${ }^{1, *}$, Xiaoping Liu², Bart Kuyken ${ }^{3}$, Richard M. Osgood, Jr. ${ }^{2}$, \\ Gunther Roelkens $^{3}$, Roel Baets ${ }^{3}$, Yurii A. Vlasov ${ }^{1}$ \\ ${ }^{1}$ IBM T. J. Watson Research Center, Yorktown Heights, NY 10598, USA \\ ${ }^{2}$ Department of Electrical Engineering, Columbia University, 1300 S. W. Mudd, 500 W. $120^{\text {th }}$ St., New York, NY 10027, USA \\ ${ }^{3}$ Photonics Research Group, Department of Information Technology, Ghent University - imec, Ghent B-9000, Belgium \\ *wgreen@us.ibm.com
}

\begin{abstract}
The silicon nanophotonic wire platform can be applied toward development of integrated optical devices for the generation and processing of mid-infrared light signals. The demonstration of a mid-infrared optical parametric amplifier will be reviewed, and recent work aimed at expanded gain bandwidth and reduced pump power requirements will be presented.
\end{abstract}

\section{INTRODUCTION}

Within the mid-infrared (mid-IR) spectral region near $\lambda=$ $2200 \mathrm{~nm}$, the silicon nanophotonic wire platform has potential to be used in constructing room temperature, highly efficient, ultra-compact devices for light generation and amplification through coherent nonlinear optical processes [1-3]. This platform could be an ideal host for a variety of mid-IR applications including molecular spectroscopy, free-space communication and environmental monitoring $[4,5]$. Strong optical confinement in silicon not only enhances the effective nonlinearity by means of reduced modal area, but also provides flexibility in the control of waveguide dispersion that is necessary for processes such as four-wave mixing (FWM) [6].

\section{MID-INFRARED OPTICAL PARAMETRIC AMPLIFIER}

We have recently demonstrated the first silicon photonic wire mid-IR optical parametric amplifier (OPA) [1]. The OPA exhibits net off-chip gain of $13 \mathrm{~dB}$ in addition to on-chip gain as large as $25.4 \mathrm{~dB}$ (Fig. 1), while having a length of only 4 $\mathrm{mm}$. By positioning the pump wavelength in the mid-IR near one-half the band-gap wavelength $(\lambda \sim 2200 \mathrm{~nm})$, the majority of parasitic TPA-related absorption is eliminated [7-9]. It is therefore possible to exploit the extremely large enhancement of the effective third-order nonlinearity $\gamma$ (Fig. 1b) afforded by high-confinement silicon photonic wire waveguides, being as much as $10^{5}$ times larger than that in conventional silica singlemode fiber [10], in order to facilitate efficient parametric processes. Moreover, waveguide dispersion engineering concepts $[6,11,12]$ have been applied to achieve a total onchip gain bandwidth exceeding $220 \mathrm{~nm}$ (Fig. 1d).

\section{BROADBAND MODULATION INSTABILITY AND RAMAN- ENHANCED FWM GAIN}

Building upon the initial mid-IR OPA demonstration described above, recent work has produced silicon wires with cross-sectional dimensions of $w=900 \mathrm{~nm}$ by $h=220 \mathrm{~nm}$,
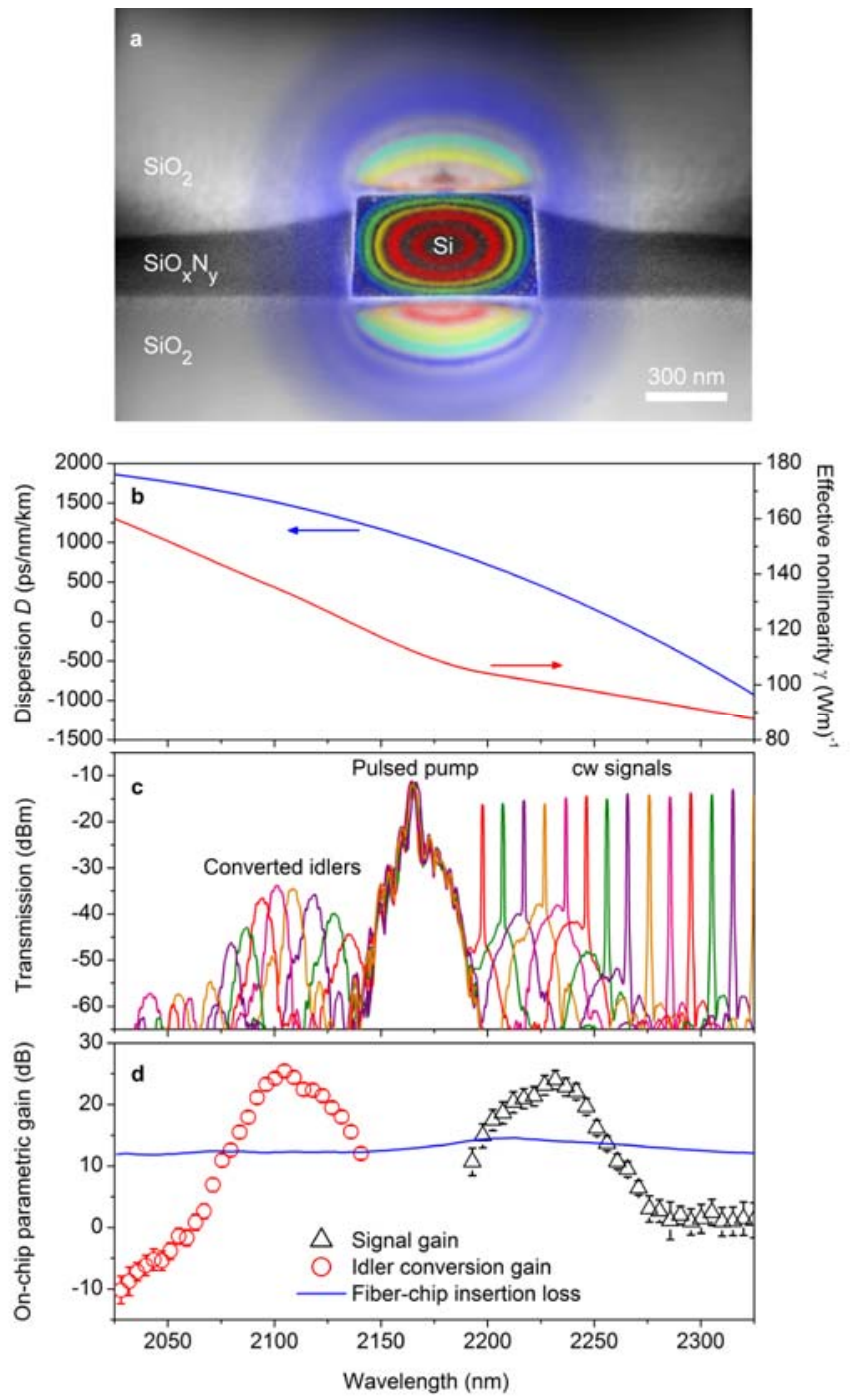

Figure 1. Engineered silicon nanophotonic waveguide characteristics, mid-IR FWM experiments, and broadband on-chip optical parametric amplification. a) SEM cross-section of the $700 \mathrm{~nm}$ x $425 \mathrm{~nm}$ silicon waveguide. The colour-map illustrates the $E_{y}$ component of the fundamental quasi-TM mode at $\lambda=2200 \mathrm{~nm}$. b) Simulated dispersion coefficient $D$ (blue curve) and effective nonlinearity parameter $y$ (red curve). c) Series of FWM spectra taken at the output of the 4 $\mathrm{mm}$-long waveguide. The peak pump power is $\mathrm{P}_{\text {pump }} \sim 27.9 \mathrm{~W}$. d) On-chip parametric signal gain (black triangles) and idler conversion gain (red circles), with bandwidth $\sim 220 \mathrm{~nm}$, and peak values $>25 \mathrm{~dB}$. Net off-chip gain results where amplification exceeds fiber-chip insertion losses (blue line), with maximum value $\sim 13 \mathrm{~dB}$. 
which are both longer $(2 \mathrm{~cm})$ to enhance the total cumulative FWM effects, as well as dispersion-optimized for ultrabroadband phase matching $[13,14]$. The wires have anomalous $\operatorname{GVD}\left(\beta_{2}<0\right)$ at wavelengths from $1800 \mathrm{~nm}$ to $2400 \mathrm{~nm}$, in addition to small positive $4^{\text {th }}$-order dispersion $\left(\beta_{4}>0\right)$ within the same range. Small $\beta_{4}$ is critical for achieving broadband phase matching and expanding the parametric gain bandwidth.

Figure 2a) illustrates that when the silicon wire is pumped with a peak input power of $\sim 13.5 \mathrm{~W}$, the output spectrum is characterized by a strong broadband modulation instability (MI) spectrum extending from $1911 \mathrm{~nm}$ to $2486 \mathrm{~nm}$. In addition, a prominent Raman Stokes peak rides on top of the MI spectrum at a wavelength of $2411 \mathrm{~nm}$, frequency downshifted from the pump by a Stokes shift of $15.6 \mathrm{THz}$ [7]. Moreover, a frequency up-shifted Raman anti-Stokes [15] peak at a wavelength of $1950 \mathrm{~nm}$ is visible. The visibility here of a strong MI background suggests that the on-chip mid-IR parametric gain available is far larger than demonstrated in previous studies [1], where MI was not observed. As illustrated in Fig. 2b), the intense MI correlates with very large values of on-chip parametric gain, exceeding $40 \mathrm{~dB}$. Moreover, we demonstrate that on-chip gain can exceed $50 \mathrm{~dB}$ in narrow bands assisted by stimulated Raman scattering. In comparison with prior work [1], the peak operating pump power is reduced to less than half. At the same time, the maximum on-chip gain obtained using the $2 \mathrm{~cm}$-long wire here shows an improvement of more than $25 \mathrm{~dB}$, while the on-chip gain bandwidth ( $>580$ $\mathrm{nm}$ ) is increased by more than $2.5 \mathrm{x}$.

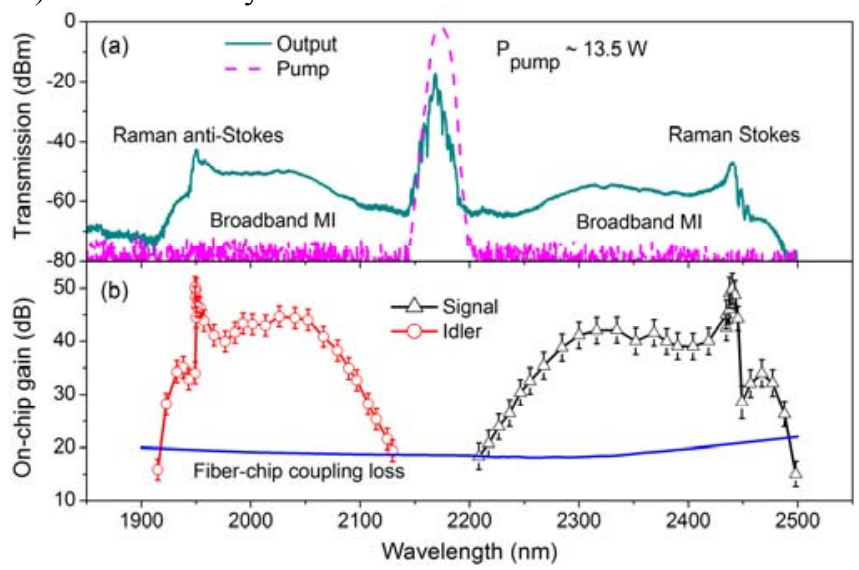

Figure 2. a) Input (dashed magenta curve) and transmitted (solid cyan curve) pump spectra, illustrating broadband MI and Raman Stokes/anti-Stokes peaks.

b) On-chip parametric gain, exhibiting broadband on-chip amplification over a bandwidth $>580 \mathrm{~nm}$, maximum Raman-assisted gain values of $\sim 50 \mathrm{~dB}$, unassisted FWM parametric gain of $\sim 40 \mathrm{~dB}$, peak net off-chip gain $>30 \mathrm{~dB}$.

\section{CONCLUSION}

These results provide a foundation for the construction of silicon-based room-temperature mid-IR light sources including tunable chip-scale parametric oscillators [16-18], optical frequency combs [19], and supercontinuum generators [20]. In this manner, silicon nanophotonic technology may be extended to an entirely new class of mid-IR applications $[4,5]$ including biochemical detection, environmental monitoring, and freespace communication, in which an integrated on-chip platform can be of significant technological benefit.

\section{REFERENCES}

[1] X. Liu, J. Osgood, R. M., Y. A. Vlasov, and W. M. J. Green, "Midinfrared optical parametric amplifier using silicon nanophotonic waveguides," Nature Photonics, vol. 4, pp. 557-560, 2010.

[2] S. Zlatanovic, J. S. Park, S. Moro, J. M. Chavez-Boggio, I. B. Divliansky, N. Alic, S. Mookherjea, and S. Radic, "Mid-infrared wavelength conversion in silicon waveguides using ultracompact telecom-band-derived pump source," Nature Photonics, vol. 4, pp. 561564, 2010.

[3] R. K. W. Lau, M. Menard, Y. Okawachi, M. A. Foster, A. C. TurnerFoster, R. Salem, M. Lipson, and A. L. Gaeta, "Mid-infrared broadband continuous-wave parametric-mixing in silicon nanowaveguides," Paper FTuC3, presented at OSA Frontiers in Optics, Rochester, NY, 2010.

[4] M. Ebrahim-Zadeh and I. T. Sorokina, Mid-Infrared Coherent Sources and Applications, 1st ed. Dordrecht: Springer, 2007.

[5] R. A. Soref, S. J. Emelett, and W. R. Buchwald, "Silicon waveguided components for the long-wave infrared region," Journal of Optics A: Pure and Applied Optics, vol. 8, pp. 840-848, 2006.

[6] X. Liu, W. M. J. Green, X. Chen, I.-W. Hsieh, J. I. Dadap, Y. A. Vlasov, and J. Osgood, R. M., "Conformal dielectric overlayers for engineering dispersion and effective nonlinearity of silicon nanophotonic wires," Optics Letters, vol. 33, pp. 2889-2891, 2008.

[7] V. Raghunathan, R. Shori, O. Stafsudd, and B. Jalali, "Nonlinear absorption in silicon and the prospects of mid-infrared silicon Raman lasers," Physica Status Solidi A, vol. 203, pp. R38-R40, 2006.

[8] A. D. Bristow, N. Rotenberg, and H. M. van Driel, "Two-photon absorption and Kerr coefficients of silicon for 850-2200 nm," Applied Physics Letters, vol. 90, pp. 191104, 2007.

[9] M. A. Foster, A. C. Turner, J. E. Sharping, B. S. Schmidt, M. Lipson, and A. L. Gaeta, "Broad-band optical parametric gain on a silicon photonic chip," Nature, vol. 441, pp. 960-963, 2006.

[10] J. I. Dadap, N. C. Panoiu, X. Chen, I.-W. Hsieh, X. Liu, C.-Y. Chou, E. Dulkeith, S. J. McNab, F. Xia, W. M. J. Green, L. Sekaric, Y. A. Vlasov, and J. Osgood, R. M., "Nonlinear-optical phase modification in dispersion-engineered Si photonic wires," Optics Express, vol. 16, pp. 1280-1299, 2008.

[11] E. Dulkeith, F. Xia, L. Schares, W. M. J. Green, and Y. A. Vlasov, "Group index and group velocity dispersion in silicon-on-insulator photonic wires," Optics Express, vol. 14, pp. 3853-3863, 2006.

[12] A. C. Turner, C. Manolatou, B. S. Schmidt, M. Lipson, M. A. Foster, J. E. Sharping, and A. L. Gaeta, "Tailored anomalous group-velocity dispersion in silicon channel waveguides," Optics Express, vol. 14, pp. 4357-4362, 2006.

[13] B. Kuyken, X. Liu, J. Osgood, R. M., Y. A. Vlasov, G. Roelkens, R. Baets, and W. M. J. Green, "Frequency conversion of mid-infrared optical signals into the telecom band using nonlinear silicon nanophotonic wires," Paper OThU4, presented at Optical Fiber Communications, Los Angeles, CA, 2010.

[14] X. Liu, B. Kuyken, G. Roelkens, R. Baets, Y. A. Vlasov, J. Osgood, R. M., and W. M. J. Green, "Mid-infrared broadband modulation instability and $50 \mathrm{~dB}$ Raman assisted parametric gain in silicon photonic wires," submitted, 2010.

[15] R. Claps, V. Raghunathan, D. Dimitropoulos, and B. Jalali, "Anti-Stokes Raman conversion in silicon waveguides," Optics Express, vol. 11, pp. 2862-2872, 2003.

[16] Q. Lin, T. J. Johnson, R. Perahia, C. P. Michael, and O. J. Painter, "A proposal for highly tunable optical parametric oscillation in silicon micro-resonators," Optics Express, vol. 16, pp. 10596-10610, 2008.

[17] J. S. Levy, A. Gondarenko, M. A. Foster, A. C. Turner-Foster, A. L. Gaeta, and M. Lipson, "CMOS-compatible multiple-wavelength oscillator for on-chip optical interconnects," Nature Photonics, vol. 4, pp. 37-40, 2010.

[18] L. Razzari, D. Duchesne, M. Ferrera, R. Morandotti, S. Chu, B. E. Little, and D. J. Moss, "CMOS compatible integrated optical hyper-parametric oscillator," Nature Photonics, vol. 4, pp. 41-45, 2010.

[19] P. Del'Haye, A. Schliesser, O. Arcizet, T. Wilken, R. Holzwarth, and T. J. Kippenberg, "Optical frequency comb generation from a monolithic microresonator," Nature, vol. 450, pp. 1214-1217, 2007.

[20] I.-W. Hsieh, X. Chen, X. Liu, J. I. Dadap, N. C. Panoiu, C.-Y. Chou, F. Xia, W. M. Green, Y. A. Vlasov, and J. Osgood, R. M., "Supercontinuum generation in silicon photonic wires," Optics Express, vol. 15, pp. 1524215249, 2007. 